\title{
Vector fields with stably limit shadowing
}

\section{Manseob Lee*}

${ }^{*}$ Correspondence:

Imsds@mokwon.ac.kr

Department of Mathematics,

Mokwon University, Daejeon,

302-729, Korea

\begin{abstract}
Let $X$ be a vector field on a closed smooth manifold $M$. In this paper, we show that if $X$ belongs to the $C^{1}$-interior of the set of all vector fields having the limit shadowing property, then it is transitive Anosov.

MSC: Primary 37C50; secondary 34D10

Keywords: hyperbolic; limit shadowing; shadowing; chain transitive; transitive;
\end{abstract} Anosov

\section{Introduction}

Discrete case dynamical system results can be extended to the case of continuous, but not always, in particular results which involve the hyperbolic structure. For instance, it is well known that if a diffeomorphism $f: M \rightarrow M$ has a $C^{1}$-neighborhood $\mathcal{U}(f)$ such that every periodic point of $g \in \mathcal{U}(f)$ is hyperbolic, then the non-wandering set $\Omega(f)$ is hyperbolic. However, the result is not true for the case of vector fields (see [1]). The notion of the limit shadowing property was introduced and studied by Eirola, Nevanlinna, and Pilyugin [2, 3]. It is different from the shadowing property (see [3]). Various shadowing properties are used in the investigation of the orbit structure. For instance, Sakai [4] and Robinson [5] proved that a diffeomorphism is structurally stable if and only if it belongs to the set of all diffeomorphisms having the shadowing property. For vector fields, Lee and Sakai [6] proved that if a vector field does not admit singularities, then the $C^{1}$-interior of the set of all vector fields having the shadowing property coincides with the set of structurally stable vector fields. Recently, in [7], Pilyugin showed that a diffeomorphism belongs to the set of all diffeomorphisms having the limit shadowing property if and only if it is $\Omega$-stable, that is, Axiom A and the no-cycle condition. From this, Carvalho [8] proved that the $C^{1}$-interior of the limit shadowing property is equal to the set of transitive Anosov diffeomorphisms. Very recently, Ribeiro [9] proved that for $C^{1}$-generic vector fields, if a vector field has the limit shadowing property in a closed isolated set, then it is a transitive hyperbolic set. Moreover, if the closed set is the whole space, then it is a transitive Anosov flow. In this result, we study the $C^{1}$-interior of the set of all vector fields having the limit shadowing property, which extends the result of [8].

Let $M$ be a closed $n(\geq 3)$-dimensional smooth Riemmanian manifold, and let $d$ be the distance on $M$ induced from a Riemannian metric $\|\cdot\|$ on the tangent bundle $T M$, and denote by $\mathfrak{X}(M)$ the set of $C^{1}$-vector fields on $M$ endowed with the $C^{1}$-topology. Then every $X \in \mathfrak{X}(M)$ generates a $C^{1}$-flow $X_{t}: M \times \mathbb{R} \rightarrow M$; that is, a $C^{1}$-map such that $X_{t}$ : $M \rightarrow M$ is a diffeomorphism satisfying $X_{0}(x)=x$ and $X_{t+s}(x)=X_{t}\left(X_{s}(x)\right)$ for all $s, t \in \mathbb{R}$ and $x \in M$.

(0) 2013 Lee; licensee Springer. This is an Open Access article distributed under the terms of the Creative Commons Attribution License (http://creativecommons.org/licenses/by/2.0), which permits unrestricted use, distribution, and reproduction in any medium, provided the original work is properly cited. 
For any $\delta>0$, a sequence $\left\{\left(x_{i}, t_{i}\right): x_{i} \in M, t_{i} \geq 1\right.$, and $\left.-\infty \leq a<i<b \leq \infty\right\}$ is a $\delta$-pseudo orbit of $X$ (or $\delta$-chain of $X$ ) if $d\left(X_{t_{i}}\left(x_{i}\right), x_{i+1}\right)<\delta$ for any $a \leq i \leq b-1$.

For the sequence $\left\{t_{i}\right\}_{i \in \mathbb{Z}}$, we denote

$$
S_{i}= \begin{cases}t_{0}+t_{1}+\cdots+t_{i-1} & \text { if } i>0, \\ 0 & \text { if } i=0, \\ -t_{-1}-t_{-2}-\cdots-t_{i} & \text { if } i<0\end{cases}
$$

We say that $X$ has the shadowing property if for any $\epsilon>0$, there is $\delta>0$ satisfying the following property: given any $\delta$-pseudo orbit $\left\{\left(x_{i}, t_{i}\right): t_{i} \geq 1, i \in \mathbb{Z}\right\}$ for all $i \in \mathbb{Z}$, there is a point $y \in M$ and an increasing homeomorphism $h: \mathbb{R} \rightarrow \mathbb{R}$ such that

$$
d\left(X_{h(t)}(y), X_{t-S_{i}}\left(x_{i}\right)\right)<\epsilon
$$

for any $i \in \mathbb{Z}$ and for any $S_{i} \leq t<S_{i+1}$.

A sequence $\left\{\left(x_{i}, t_{i}\right): t_{i} \geq 1, i \in \mathbb{Z}\right\}$ is a limit pseudo orbit of $X$ if for $t_{i} \geq 1, i \in \mathbb{Z}$,

$$
d\left(X_{t_{i}}\left(x_{i}\right), x_{i+1}\right) \rightarrow 0 \quad \text { as } i \rightarrow \pm \infty
$$

The following definition introduced by Yujin et al. [10] is different from the notion of Ribeiro [9]. We say that $X$ has the limit shadowing property if for any $\left\{\left(x_{i}, t_{i}\right): t_{i} \geq 1, i \in \mathbb{Z}\right\}$, there is $y \in M$ and an increasing homeomorphism $h: \mathbb{R} \rightarrow \mathbb{R}$ with $h(0)=0$ such that for $S_{i} \leq t<S_{i+1}$,

$$
d\left(X_{h(t)}(y), X_{t-S_{i}}\left(x_{i}\right)\right) \rightarrow 0 \quad \text { as } i \rightarrow \pm \infty
$$

Denote by $\mathcal{L} \mathcal{S P}(M)$ the set of all diffeomorphisms having the limit shadowing property. We say that $X$ has the $C^{1}$-stably limit shadowing property if there is a $C^{1}$-neighborhood $\mathcal{U}(X)$ of $X$ such that for any $Y \in \mathcal{U}(X), Y$ has the limit shadowing property.

Let $\Lambda$ be an $X_{t}$-invariant compact set. The $\Lambda$ is called hyperbolic for $X_{t}$ if there are constants $C>0, \lambda>0$ and a splitting $T_{x} M=E_{x}^{s} \oplus\langle X(x)\rangle \oplus E_{x}^{u}$ such that the tangent flow $D X_{t}: T M \rightarrow T M$ leaves invariant the continuous splitting and

$$
\left\|\left.D X_{t}\right|_{E_{x}^{s}}\right\| \leq C e^{-\lambda t} \quad \text { and } \quad\left\|\left.D X_{-t}\right|_{E_{x}^{u}} ^{u}\right\| \leq C e^{-\lambda t}
$$

for $t>0$ and $x \in \Lambda$. If $\Lambda=M$, then $X$ is Anosov.

A point $x \in M$ is called non-wandering of $X_{t}$ if for any neighborhood $U$ of $x$, there is $t \geq 1$ such that $X_{t}(U) \cap U \neq \emptyset$. The set of non-wandering points of $X$ is denoted by $\Omega(X)$. Then we know that $\operatorname{Sing}(X) \cup P(X) \subset \Omega(X)$. Here $\operatorname{Sing}(X)$ is the set of singularities of $X$, and $P(X)$ is the set of periodic orbits of $X$. Define $\operatorname{Crit}(X)=\operatorname{Sing}(X) \cup P(X)$. Here, for any $\sigma \in \operatorname{Crit}(X), \sigma$ is called the critical element of $X$. We say that $X \in \mathfrak{X}(M)$ is transitive if there is a point $x \in M$ such that $\omega_{X}(x)=M$, where $\omega_{X}(x)$ is the omega limit set of $x$. The following is the main result of this paper.

Theorem 1.1 Let $X \in \mathfrak{X}(M)$. If $X$ belongs to the $C^{1}$-interior of $\mathcal{L} \mathcal{S P}(M)$, then $X$ is transitive Anosov. 


\section{Proof of Theorem 1.1}

Let $M$ be as before, and let $X \in \mathfrak{X}(M)$. We say that a vector field $X \in \mathfrak{X}(M)$ is robustly transitive if there exists a $C^{1}$-neighborhood $\mathcal{U}(X)$ of $X$ such that for all $Y \in \mathcal{U}(X), Y$ is transitive. For robustly transitive vector fields, Doering [11] showed that if a compact manifold $M$ is three-dimensional, then every robustly transitive vector field is Anosov. In [12], Vivier proved that robustly transitive flows on the whole $n$-dimensional compact without boundary manifold $M$ must have a dominated splitting for the linear Poincaré flow and have no singularities.

Theorem 2.1 [12, Theorem 1] If $X$ is a robustly transitive vector field, then $X$ admits no singular points.

We say that a vector field $X \in \mathfrak{X}(M)$ is homogeneous on $U \subset M$ if there is a $C^{1}$-neighborhood $\mathcal{U}(X)$ of $X$ such that

(a) for all $Y \in \mathcal{U}(X)$, there are no sinks nor sources in $U$,

(b) every critical element of $Y$ in $\Lambda_{Y}(U)=\bigcap_{t \in \mathbb{R}} Y_{t}(U)$ is hyperbolic, and

(c) the index of the continuation on $\mathcal{U}(X)$ of every critical point does not change.

From the definition, we know the following theorem.

Theorem 2.2 [13, Theorem 4.1] Let $X \in \mathfrak{X}(M)$ be a robustly transitive homogeneous flow on an $n(\geq 3)$-manifold $M$. Then $X$ is Anosov.

Let $\Lambda$ be a closed $X_{t}$-invariant set. We say that $\Lambda$ is attracting if $\Lambda=\bigcap_{t \geq 0} X_{t}(U)$ for some neighborhood $U$ of $\Lambda$ satisfying $X_{t}(\bar{U}) \subset U$ for all $t>0$. An attractor of $X$ is a transitive attracting set of $X$ and a repeller is an attractor for $-X$. We say that $\Lambda$ is a proper attractor or proper repeller if $\emptyset \neq \Lambda \neq M$.

Lemma 2.3 [9, Proposition 3] A vector field $X$ is chain transitive in an isolated set $\Lambda$ if and only if $\Lambda$ has no proper attractor for $X$.

By Lemma 2.3, if $\Lambda=M$, then we omit the isolated condition. Then we have the following.

Lemma 2.4 If $X$ has the limit shadowing property, then $X$ has no proper attractor.

Proof To derive a contradiction, we may assume that $X$ has a proper attractor $\Lambda$. By definition, $\Lambda \neq \emptyset$ and $\Lambda \neq M$. Since $\Lambda$ is an attractor, there is $\eta>0$ such that $\Lambda \subset B_{\eta}(\Lambda)$, where $B_{\eta}(\Lambda)$ is the $\eta$-neighborhood of $\Lambda$. Since $M \backslash \Lambda \neq \emptyset$, choose $x \in M \backslash B_{\eta}(\Lambda)$ and $y \in \Lambda$. Then we can construct a limit pseudo orbit $\left\{\left(x_{i}, t_{i}\right): t_{i} \geq 1, i \in \mathbb{Z}\right\}$ as follows: for all $i \in \mathbb{Z}$, (i) $X_{i}(x)=x_{i}, t_{i}=1, i>0$ and (ii) $X_{i}(y)=x_{i}, t_{i}=1, i \leq 0$. By the limit shadowing property, there are $z \in M$ and an increasing homeomorphism $h: \mathbb{R} \rightarrow \mathbb{R}$ with $h(0)=0$ such that for $i \in \mathbb{Z}, d\left(X_{h(t)}(z), X_{t-S_{i}}\left(x_{i}\right)\right) \rightarrow 0$ as $i \rightarrow \pm \infty$. Then we can find $-k \in \mathbb{N}$ such that

$$
d\left(X_{h(t)}(z), X_{t-S_{k}}\left(x_{-k}\right)\right)=d\left(X_{h(t)}(z), X_{t-S_{k}}\left(X_{-k}(y)\right)<\frac{\eta}{16}\right.
$$


for $S_{k} \leq t<S_{k+1}$. Thus, for all $S_{k} \leq t<S_{k+1}, X_{t}\left(X_{h(t)}(z)\right) \in B_{\eta}(\Lambda)$ for all $t \geq 0$. Since $B_{\eta}(\Lambda)$ is an attracting neighborhood of $\Lambda$, for $S_{k} \leq t<S_{k+1}$,

$$
\bigcap_{t \geq 0} X_{t}\left(X_{h(t)}(z)\right) \in \bigcap_{t \geq 0}\left(B_{\eta}(\Lambda)\right)=\Lambda
$$

Since $\Lambda$ is an $X_{t}$-invariant set, we know that $z \in \Lambda$. Thus we have that

$$
d\left(X_{t}(z), X_{t}(x)\right)>\eta \quad \text { for all } t>0 \text {. }
$$

This is a contradiction.

We introduce the notion of chain transitive set, which is a weaker notion of transitive set. We say that a set $\Lambda$ is chain transitive if for any $x, y \in \Lambda$ and $\delta>0$, there is a $\delta$-pseudo orbit $\left\{x_{0}=x, x_{1}, \ldots, x_{n}=y\right\}$ with $x_{i} \in \Lambda$ for any $t_{i} \geq 1$ and $0 \leq i \leq n$. The following is a version of the vector fields of the result of Gu [14].

Lemma 2.5 [14, Theorem 4.1] Let $X \in \mathfrak{X}(M)$. If $X$ has the limit shadowing property, then $X$ is transitive if and only if $X$ is chain transitive.

By Lemma 2.5, $X$ is transitive, $X$ does not contain sinks or sources. Thus, for $\gamma \in P(X)$, we just consider saddle-type periodic orbits. Let $\gamma$ be a hyperbolic closed orbit of a vector field $X \in \mathfrak{X}(M)$. We define the stable and unstable manifolds of $\gamma$ by

$$
W^{s}(\gamma)=\left\{y \in M: \omega_{X}(y)=\gamma\right\} \text { and } W^{u}(\gamma)=\left\{y \in M: \alpha_{X}(y)=\gamma\right\} \text {. }
$$

Denote by index $(\gamma)$ the dimension of the stable manifold of $\gamma$.

Lemma 2.6 Let $\eta, \gamma \in P(X)$ be hyperbolic orbits. If $X$ has the limit shadowing property, then $W^{s}(\eta) \cap W^{u}(\gamma) \neq \emptyset$.

Proof Let $\eta, \gamma \in P(X)$ be hyperbolic orbits. Suppose that $X$ has the limit shadowing property. Take $p \in \eta$ and $q \in \gamma$ such that $X_{\pi(p)}(p)=p$ and $X_{\pi(q)}(q)=q$, where $\pi(a)$ is the period of $a$. For $i \in \mathbb{Z}$, put $X_{i}(p)=x_{i}, t_{i}=1, i \leq 0$, and $X_{i}(q)=x_{i+1}, t_{i}=1, i \geq 0$. Then

$$
\left\{\left(x_{i}, t_{i}\right): t_{i}=1, i \in \mathbb{Z}\right\}=\left\{\ldots, x_{-1}, x_{0}(=p), x_{1}(=q), x_{2}, \ldots\right\}
$$

is a limit pseudo orbit of $X$. By the limit shadowing property, there are $y \in M$ and an increasing homeomorphism $h: \mathbb{R} \rightarrow \mathbb{R}$ with $h(0)=0$ such that for $S_{i} \leq t<S_{i+1}$, $d\left(X_{h(t)}(y), X_{t-S_{i}}\left(x_{i}\right)\right) \rightarrow 0$ as $i \rightarrow \pm \infty$. Then we can find $j \in \mathbb{Z}$ such that for $-j \leq t<-j+1$,

$$
X_{t-S_{j}}\left(x_{j}\right)=X_{t-(-j)}\left(X_{-j}(p)\right)=X_{t}(p) .
$$

Then, for $S_{j} \leq t<S_{j+1}$, we have $d\left(X_{h(t)}(y), X_{t-S_{j}}\left(x_{j}\right)\right)=d\left(X_{h(t)}(y), X_{t}(p)\right)$. If $t \rightarrow-\infty$, then

$$
d\left(X_{t}(y), X_{t}(p)\right)=d\left(X_{t}(y), \eta\right) \rightarrow 0
$$


Also, we can find $k \in \mathbb{Z}$ such that for $k \leq t<k+1$,

$$
X_{t-S_{k}}\left(x_{k}\right)=X_{t-k}\left(X_{k-1}(q)\right)=X_{t-1}(q) .
$$

Then, for $S_{k} \leq t<S_{k+1}$, we have $d\left(X_{h(t)}(y), X_{t-S_{k}}\left(x_{k}\right)\right)=d\left(X_{h(t)}(y), X_{t-1}(p)\right)$. If $t \rightarrow \infty$, then

$$
d\left(X_{t}(y), X_{t}(p)\right)=d\left(X_{t}(y), \gamma\right) \rightarrow 0 .
$$

Thus $\mathcal{O}_{X}(y) \cap W^{u}(\eta) \cap W^{s}(\gamma)$, and so $W^{u}(\eta) \cap W^{s}(\gamma) \neq \emptyset$, where $\mathcal{O}_{X}(y)$ is the orbits of $y$.

A vector field $X \in \mathfrak{X}(M)$ is Kupka-Smale if its critical orbits are all hyperbolic and their stable and unstable manifolds intersect transversely. It is well known that the Kupka-Smale vector field is a residual subset in $\mathfrak{X}(M)$. Denote by $\mathcal{K} \mathcal{S}(M)$ the set of all vector fields satisfying the Kupka-Smale.

Lemma 2.7 [15, Lemma 3.4] Let $X \in \mathfrak{X}(M)$ be a Kupka-Smale vector field, and let $\eta, \gamma \in$ $P(X)$ be hyperbolic orbits. If $\operatorname{dim} W^{s}(\eta)+\operatorname{dim} W^{u}(\gamma) \leq \operatorname{dim} M$, then $W^{s}(\eta) \cap W^{u}(\gamma)=\emptyset$.

Lemma 2.8 Let $X$ have the $C^{1}$-stably limit shadowing property, and let $\mathcal{U}(X)$ be as in the definition. Then, for any $Y \in \mathcal{U}(X)$ and for any hyperbolic $\eta, \gamma \in P(Y)$, index $(\eta)=\operatorname{index}(\gamma)$.

Proof Suppose that $X$ has the $C^{1}$-stably limit shadowing property. Then there is a $C^{1}$-neighborhood $\mathcal{U}(X)$ of $X$ such that for any $Y \in \mathcal{U}(X), Y$ has the limit shadowing property. Let $\eta, \gamma \in P(Y)$ be hyperbolic orbits. To derive a contradiction, we may assume that $\operatorname{index}(\eta) \neq \operatorname{index}(\gamma)$. Then we know that $\operatorname{dim} W^{s}(\eta)+\operatorname{dim} W^{u}(\gamma)<\operatorname{dim} M$ or $\operatorname{dim} W^{u}(\eta)+\operatorname{dim} W^{s}(\gamma)<\operatorname{dim} M$. Assume that $\operatorname{dim} W^{s}(\eta)+\operatorname{dim} W^{u}(\gamma)<\operatorname{dim} M$. Since $X$ has the $C^{1}$-stably limit shadowing property, we can choose $Z \in \mathcal{V}(Y) \subset \mathcal{U}(X) \cap \mathcal{K} \mathcal{S}(M)$ such that $\operatorname{dim} W^{s}\left(\eta_{Z}\right)+\operatorname{dim} W^{u}\left(\gamma_{Z}\right)<\operatorname{dim} M$, where $\eta_{Z}$ and $\gamma_{Z}$ are the continuations of $\eta$ and $\gamma$, respectively. Since $Z \in \mathcal{K} \mathcal{S}(M)$, by Lemma $2.7, W^{s}\left(\eta_{Z}\right) \cap W^{u}\left(\gamma_{Z}\right)=\emptyset$. Since $Z$ has the limit shadowing property, by Lemma 2.6 this is a contradiction.

Lemma 2.9 [15, Theorem 4.3] Let $\mathcal{U}(X)$ be a $C^{1}$-neighborhood of $X$. If $\gamma \in P(X)$ is not hyperbolic, then there is $Y \in \mathcal{U}(X)$ such that $Y$ has two hyperbolic orbits $\eta_{1}, \eta_{2} \in P(Y)$ with different indices.

Proof of Theorem 1.1 Let $X$ have the $C^{1}$-stably limit shadowing property. Then there is a $C^{1}$-neighborhood $\mathcal{U}(X)$ of $X$ such that for any $Y \in \mathcal{U}(X), Y$ has the limit shadowing property. To get the conclusion, it is enough to show that every $\gamma \in P(Y)$ is hyperbolic by Lemma 2.8. By contradiction, we may assume that $\gamma \in P(Y)$ is not hyperbolic. Then, by Lemma 2.9, there is $Z \in \mathcal{U}(X)$ such that $Z$ has two hyperbolic periodic orbits $\eta_{1}, \eta_{2} \in P(Z)$ with different indices. Since $Z$ has the limit shadowing property, this is a contradiction by Lemma 2.8 . 
Author's contributions

The author carried out the proof of the theorem and approved the final manuscript.

\section{Acknowledgements}

We thank the referees very much for their helpful comments and suggestions. The second author was supported by the Basic Science Research Program through the National Research Foundation of Korea (NRF) funded by the Ministry of Education, Science and Technology (No. 2011-0007649).

\section{Received: 10 June 2013 Accepted: 8 August 2013 Published: 21 August 2013}

\section{References}

1. Gan, S, Wen, L: Nonsingular star flows satisfy Axiom A and the no-cycle condition. Invent. Math. 164, 279-315 (2006)

2. Eirola, T, Nevanlinna, O, Pilyugin, S: Limit shadowing property. Numer. Funct. Anal. Optim. 18, 75-92 (1997)

3. Pilyugin, SY: Shadowing in Dynamical Systems. Lecture Notes in Math., vol. 1706. Springer, Berlin (1999)

4. Sakai, K: Pseudo orbit tracing property and strong transversality of diffeomorphisms on closed manifolds. Osaka J. Math. 31, 373-386 (1994)

5. Robinson, C: Stability theorems and hyperbolicity in dynamical systems. Rocky Mt. J. Math. 7, 425-437 (1977)

6. Lee, K, Sakai, K: Structurally stability of vector fields with shadowing. J. Differ. Equ. 232, 303-313 (2007)

7. Pilyugin, SY: Sets of dynamical systems with various limit shadowing properties. J. Dyn. Differ. Equ. 19, 747-775 (2007)

8. Carvalho, B: Hyperbolicity, transitivity and the two-sided limit shadowing property. arXiv:1301.2356v1

9. Ribeiro, R: Hyperbolicity and types of shadowing for $C^{1}$-generic vector fields. arXiv:1305.2977v1

10. Yujun, Z, Jilian, Z, Yanping, G: Invariant properties of limit shadowing. Appl. Math. J. Chin. Univ. Ser. B 19, 279-287 (2004)

11. Doering, C: Persistently transitive vector fields on three manifolds. In: Dynam. Syst. Biff. Theory. Pitman Res. Notes, vol. 160, pp. 59-89 (1987)

12. Vivier, T: Projective hyperbolicity and fixed points. Ergod. Theory Dyn. Syst. 26, 923-936 (2006)

13. Araujo, V, Pacifico, M: Three-Dimensional Flows. Springer, Berlin (2010)

14. Gu, R: Recurrence and the asymptotic pseudo-orbit tracing property. Nonlinear Anal. 66, 1698-1706 (2007)

15. Arbieto, A, Senos, L, Sodero, T: The specification property for flows from the robust and generic view point. J. Differ. Equ. 253, 1893-1909 (2012)

doi:10.1186/1687-1847-2013-255

Cite this article as: Lee: Vector fields with stably limit shadowing. Advances in Difference Equations 2013 2013:255.

\section{Submit your manuscript to a SpringerOpen ${ }^{\circ}$ journal and benefit from:}

- Convenient online submission

- Rigorous peer review

- Immediate publication on acceptance

- Open access: articles freely available online

- High visibility within the field

- Retaining the copyright to your article 